\title{
On the Possibility of the Jerk Derivative in Electrical Circuits
}

\author{
J. F. Gómez-Aguilar, ${ }^{1}$ J. Rosales-García, ${ }^{2}$ R. F. Escobar-Jiménez, ${ }^{3}$ M. G. López-López, ${ }^{3}$ \\ V. M. Alvarado-Martínez, ${ }^{3}$ and V. H. Olivares-Peregrino ${ }^{3}$ \\ ${ }^{1}$ CONACYT-Centro Nacional de Investigación y Desarrollo Tecnológico, Tecnológico Nacional de México, \\ Interior Internado Palmira S/N, Col. Palmira, 62490 Cuernavaca, MOR, Mexico \\ ${ }^{2}$ Departamento de Ingeniería Electrica, DICIS, Universidad de Guanajuato, Carretera Salamanca-Valle de Santiago, \\ Km. $3.5+1.8$ Km., Comunidad de Palo Blanco, Salamanca, GTO, Mexico \\ ${ }^{3}$ Centro Nacional de Investigación y Desarrollo Tecnológico, Tecnológico Nacional de México, Interior Internado Palmira S/N, \\ Col. Palmira, 62490, Cuernavaca, MOR, Mexico
}

Correspondence should be addressed to J. F. Gómez-Aguilar; jgomez@ier.unam.mx

Received 28 June 2016; Revised 15 August 2016; Accepted 22 September 2016

Academic Editor: Alexander Iomin

Copyright (C) 2016 J. F. Gómez-Aguilar et al. This is an open access article distributed under the Creative Commons Attribution License, which permits unrestricted use, distribution, and reproduction in any medium, provided the original work is properly cited.

A subclass of dynamical systems with a time rate of change of acceleration are called Newtonian jerky dynamics. Some mechanical and acoustic systems can be interpreted as jerky dynamics. In this paper we show that the jerk dynamics are naturally obtained for electrical circuits using the fractional calculus approach with order $\gamma$. We consider fractional LC and RL electrical circuits with $1 \leqslant \gamma<2$ for different source terms. The LC circuit has a frequency $\omega$ dependent on the order of the fractional differential equation $\gamma$, since it is defined as $\omega(\gamma)=\omega_{0}^{\gamma} \gamma^{1-\gamma}$, where $\omega_{0}$ is the fundamental frequency. For $\gamma=3 / 2$, the system is described by a third-order differential equation with frequency $\omega \sim \omega_{0}^{3 / 2}$, and assuming $\gamma=2$ the dynamics are described by a fourth differential equation for jerk dynamics with frequency $\omega \sim \omega_{0}^{2}$.

\section{Introduction}

Fractional calculus (FC) generalizes integer order derivatives and integrals; the mathematical formalisms were developed by Euler, Abel, Fourier, Liouville, Riemann, Grünwald, and Riesz, among many others [1-4]. The utility of the fractional derivatives and the fractional integrals resides on their feasibility for describing nonlocal properties because these consider the history and the nonlocal distributed effects of any physical system [5-8]. The classical electrical circuits consisting of resistors, capacitors, and inductors are conventionally described by integer order models. However, the electrical components have a nonconservative behavior, since they involve irreversible dissipative effects such as ohmic friction or internal friction; additionally, these components entail thermal memory and nonlinearities due to the effects of the electric and magnetic fields $[9,10]$. The FC is applied to a variety of electrical circuit problems, such as domino ladders and tree structures, and to study a number of elements (coils, memristor, etc.) [11-15]. The use of fractional order operators allows us to generalize the propagation of electrical signals in devices, circuits, and networks [16-21].

With a basis on the fractional calculus concepts and previous works developed by the authors [22-27], this paper aims to describe fractional higher order power dissipation in resistive elements. This higher noninteger dynamics are known as the Newtonian jerky dynamics. These dynamics have been of interest in certain applications of mechanics, acoustics, and electrical circuits [28-37].

Here it is shown that if we have a fractional differential equation describing some physical process, then it is relatively easy to obtain the fractional jerky dynamics due the fractional order derivative $n-1<\gamma<n$. We consider fractional LC and RL electrical circuits with $1 \leqslant \gamma<2$ for different source terms. In the case of LC circuits the frequency $\omega$ is in general proportional to the fractional exponent of the fundamental frequency $\omega_{0}$. 


\section{Basic Concepts on Fractional Calculus}

The Caputo fractional derivative ${ }_{0}^{C} D_{t}^{\gamma}$ for a function $f(t)$ is defined as [3]

$$
{ }_{0}^{C} D_{t}^{\gamma} f(t)=\frac{1}{\Gamma(n-\gamma)} \int_{0}^{t} \frac{f^{(n)}(\eta)}{(t-\eta)^{\gamma-n+1}} d \eta
$$

$$
n-1 \leqslant \gamma<n,
$$

where $n=1,2, \ldots \in \mathbb{N}$ and $\gamma$ is the order of the fractional derivative. From (1), it follows that the derivative of a constant is zero and the initial conditions of the fractional order differential equations are the same ones needed by the ordinary differential equations with a known physical interpretation [1].

The Laplace transform of the Caputo derivative (1) has the form [3]

$$
\begin{aligned}
\mathscr{L}\left[{ }_{0}^{C} D_{t}^{\gamma} f(t)\right]=s^{\gamma} F(s)-\sum_{k=0}^{n-1} s^{\gamma-k-1} f^{(k)}(0), & \\
& n-1<\gamma \leqslant n,
\end{aligned}
$$

where $F(s)$ is the Laplace transform of the function $f(t)$ and $n \in \mathbb{N}$. For different ranges of $\gamma$ we have the following Laplace transform, corresponding to the fractional Caputo derivative

$$
\begin{aligned}
\mathscr{L}\left[{ }_{0}^{C} D_{t}^{\gamma} f(t)\right] & =s^{\gamma} F(s)-s^{\gamma-1} f(0) \quad 0<\gamma \leqslant 1, \\
\mathscr{L}\left[{ }_{0}^{C} D_{t}^{\gamma} f(t)\right] & =s^{\gamma} F(s)-s^{\gamma-1} f(0)-s^{\gamma-2} f^{\prime}(0) \\
1 & <\gamma \leqslant 2 .
\end{aligned}
$$

The Mittag-Leffler function is defined as a power series [3]

$$
E_{\gamma}(z)=\sum_{n=0}^{\infty} \frac{z^{n}}{\Gamma(\gamma n+1)} \quad \Re(\gamma)>0,
$$

where $\mathfrak{R}$ indicates the real part; in general, $z$ is a complex quantity. For $\gamma=1$, we have $e^{z}$; therefore, the Mittag-Leffler function is a generalization of the exponential function. The generalization of (5) is given by

$$
E_{\gamma, \beta}(z)=\sum_{n=0}^{\infty} \frac{z^{n}}{\Gamma(\gamma n+\beta)} \quad \mathfrak{R}(\gamma)>0, \mathfrak{R}(\beta)>0,
$$

where $\Gamma(\cdot)$ is the Gamma Euler function. The Laplace transform of the function $t^{\beta-1} E_{\gamma, \beta}\left(a t^{\gamma}\right)$ is

$$
\int_{0}^{\infty} e^{-s t} t^{\beta-1} E_{\gamma, \beta}\left( \pm a t^{\gamma}\right) d t=\frac{s^{\gamma-\beta}}{s^{\gamma} \mp a},
$$

where $a$ is a constant. Consequently, the inverse Laplace transform is given by

$$
\mathscr{L}^{-1}\left[\frac{s^{\gamma-\beta}}{s^{\gamma} \mp a}\right]=t^{\beta-1} E_{\gamma, \beta}\left( \pm a t^{\gamma}\right) .
$$

From (6) we have some common Mittag-Leffler functions [3]

$$
\begin{aligned}
E_{1,1}( \pm z) & =e^{ \pm z} \\
E_{2,1}\left(-z^{2}\right) & =\cos (z) .
\end{aligned}
$$

\section{Electrical Circuits}

3.1. LC Electrical Circuit. A systematic way to construct fractional differential equations is given in [22]. In [23] it was shown that fractional LC circuits with constant source $V_{0}$ can be described by the equation

$$
\frac{L}{\sigma^{2(1-\gamma)}} \frac{d^{2 \gamma} q(t)}{d t^{2 \gamma}}+\frac{q(t)}{C}=V_{0}, \quad 1<\gamma \leqslant 2 .
$$

To be consistent with the dimensionality the parameter $\sigma$ has dimensions of seconds. The parameter $\sigma$ characterizes the fractional temporal structures giving an intermediate behavior between a conservative and dissipative system. The corresponding differential equations are of noninteger order and are related with a fractal space-time geometry. Therefore we have a new family of solutions [22]. The derivative considered is the Caputo type of order $\gamma$ given by (1). The case with $n=1$ for $0<\gamma \leqslant 1$ was reported in [26].

In this work we study the case when $n=2$ and $1<\gamma \leqslant 2$, using the Caputo derivative for LC and RL electrical circuits. We can rewrite (11) as

$$
\frac{d^{2 \gamma} q}{d t^{2 \gamma}}+\omega^{2} q=A, \quad 1<\gamma \leqslant 2,
$$

where

$$
\omega^{2}(\gamma)=\frac{\sigma^{2(1-\gamma)}}{L C}=\omega_{0}^{2} \sigma^{2(1-\gamma)}
$$

is the fractional angular frequency depending on $\gamma, \omega_{0}=$ $1 / \sqrt{L C}$ is the fundamental frequency of the system, and $A=$ $\left(\sigma^{2(1-\gamma)} / L\right) V_{0}$ is defined in terms of a constant source $V_{0}$. Due to dimensions of $\sigma$ (second), we observe that the relation between the auxiliary parameter $\sigma$ and the fractional order derivative $\gamma$ is given by [23]

$$
\gamma=\frac{\sigma}{\sqrt{L C}}, \quad 1<\sigma \leqslant 2 \sqrt{L C}
$$

In this case, (13) may be written only in terms of $\gamma$

$$
\omega(\gamma)=\omega_{0}^{\gamma} \gamma^{1-\gamma}, \quad 1<\gamma \leqslant 2
$$

if $\gamma=1$, from (12) and (15) we encounter the conventional LC circuit with frequency $\omega=\omega_{0}$, and then, for $\gamma=3 / 2$ and also from (12) and (15), the system representation becomes a third-order differential equation with frequency $\omega^{2}(3 / 2)=$ $(2 / 3) \omega_{0}^{3}$. The third time derivative is called jerk (jerky dynamic) and, by definition, it is the first time derivative of the acceleration and has already been used before for assessing the comfort of motion, for example, in designing lifts and for slowly rotating rolling bearings [29]. If $\gamma=2$, the system is described by a fourth-order differential equation with frequency $\omega(2)=\omega_{0}^{2} / 2$. Higher order derivatives permit described vibration analysis, control theory, and robotics applications [30-37]. 


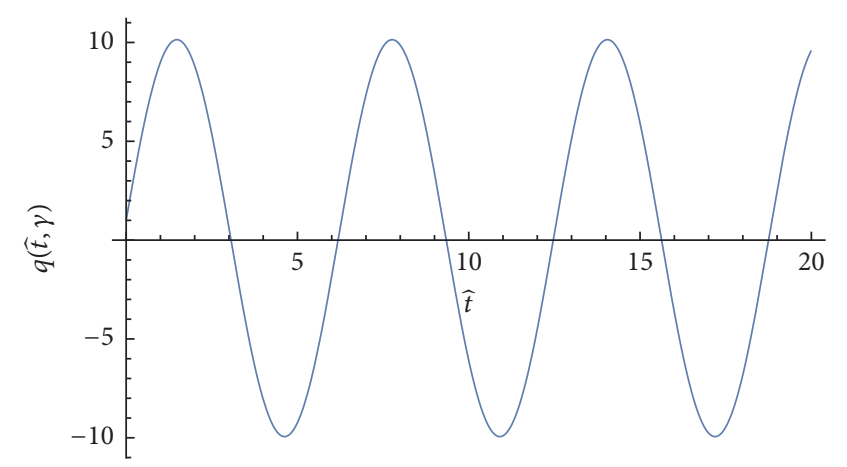

(a)

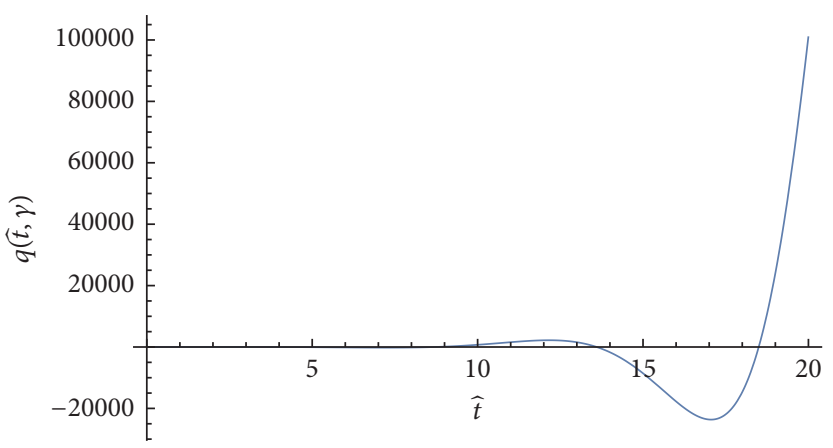

(c)

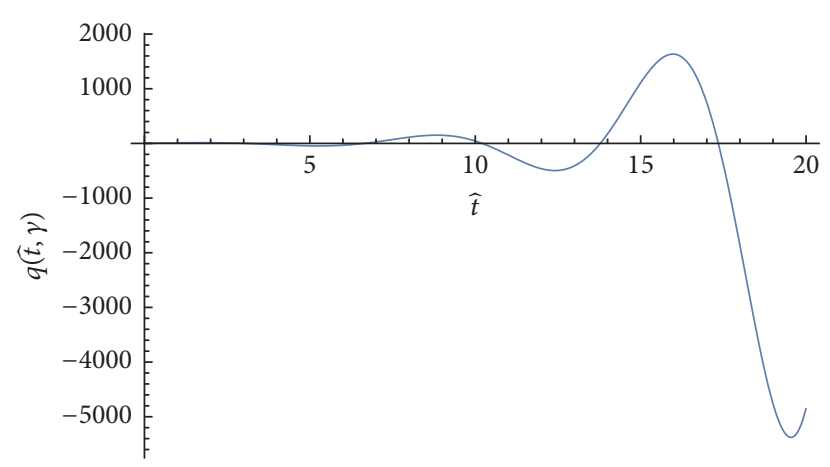

(b)

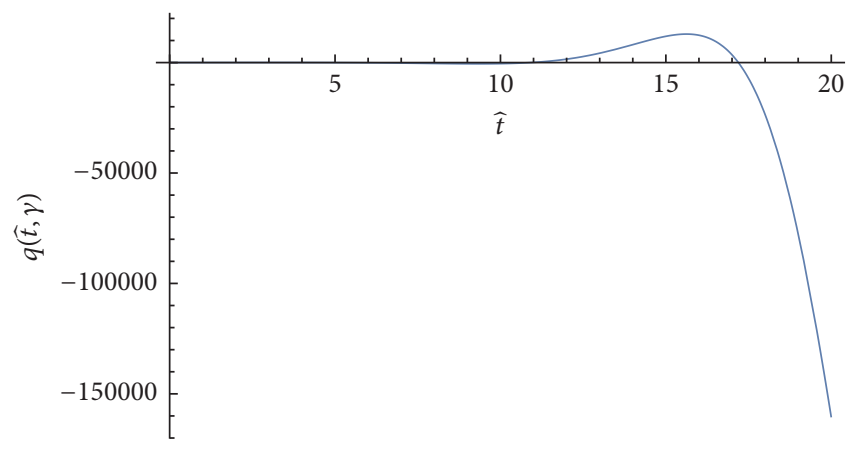

(d)

Figure 1: Simulation of (16) for (a) $\gamma=1$ (classical case); (b) $\gamma=1.3$; (c) $\gamma=1.5$; and (d) $\gamma=2.0$.

We suppose the following initial conditions $q(0)=q_{0}$ and $\dot{q}(0)=I_{0}$; then applying direct (4) and the inverse Laplace transforms to (12) we find a particular solution given by

$$
\begin{aligned}
q(\hat{t} ; \gamma)= & C V_{0}+\left(q_{0}-C V_{0}\right) E_{2 \gamma}\left(-\gamma^{2(1-\gamma)} \hat{t}^{2 \gamma}\right) \\
& +\frac{I_{0}}{\omega_{0}} \widetilde{t} E_{2 \gamma, 2}\left(-\gamma^{2(1-\gamma)} \hat{t}^{2 \gamma}\right) \quad 1<\gamma \leqslant 2,
\end{aligned}
$$

where $\widehat{t}=\omega_{0} t$ is a dimensionless parameter. For $\gamma=1$ we have

$$
q(t ; 1)=C V_{0}+\left(q_{0}-C V_{0}\right) \cos \omega_{0} t+\frac{I_{0}}{\omega_{0}} \sin \left(\omega_{0} t\right),
$$

which is the well-known result for the conventional case. Consider the electrical circuit LC with $L=10 \mathrm{H}, C=0.1 \mathrm{~F}$, and $V(0)=10$ volts. Plots for values of $\gamma$ within $1<\gamma \leqslant 2$ are represented in Figures 1(a), 1(b), 1(c), and 1(d).

Suppose now that we have an alternating source; thus

$$
\frac{d^{2 \gamma} q}{d t^{2 \gamma}}+\omega^{2} q=A \cos \omega_{p} t \quad 1<\gamma \leqslant 2,
$$

where $A=\left(\sigma^{2(1-\gamma)} / L\right) V_{0}, \omega$ is given by $(15)$, and $\omega_{p}$ is the frequency of the source. Applying Laplace transform (4) with the initial conditions $q(0)=q_{0}$ and $\dot{q}(0)=I_{0}$ we have

$$
\begin{aligned}
Q(s)= & q_{0} \frac{s^{2 \gamma-1}}{s^{2 \gamma}+\omega^{2}}+I_{0} \frac{s^{2 \gamma-2}}{s^{2 \gamma}+\omega^{2}} \\
& +A \frac{s}{\left(s^{2 \gamma}+\omega^{2}\right)\left(s^{2}+\omega_{p}^{2}\right)},
\end{aligned}
$$

for the third term in (19), we select the highest power of $s$ as a common factor from the denominator, and then we expand the denominator in an alternating geometric series [38]; as a result we have

$$
\begin{aligned}
Q(s)= & q_{0} \frac{s^{2 \gamma-1}}{s^{2 \gamma}+\omega^{2}}+I_{0} \frac{s^{2 \gamma-2}}{s^{2 \gamma}+\omega^{2}} \\
& +A \sum_{m, n=0}^{\infty}(-1)^{m+n} \frac{\omega^{2 m} \omega_{p}^{2 n}}{s^{2 \gamma(n+1)+2 m+1}} .
\end{aligned}
$$

Applying the inverse Laplace transform, finally, the solution is

$$
\begin{aligned}
& q(t ; \gamma)=q_{0} E_{2 \gamma}\left[-\gamma^{2(1-\gamma)} \omega_{0}^{2 \gamma} t^{2 \gamma}\right] \\
& \quad+I_{0} t E_{2 \gamma, 2}\left(-\gamma^{2(1-\gamma)} \omega_{0}^{2 \gamma} t^{2 \gamma}\right) \\
& \quad+C V_{0} \sum_{m, n=0}^{\infty} \frac{(-1)^{m+n} \omega_{p}^{2 m} \gamma^{2(1-\gamma)(n+1)} \omega_{0}^{2(n+1) \gamma}}{\Gamma[2 \gamma(n+1)+2 m+1]} t^{2 \gamma(n+1)+2 m} .
\end{aligned}
$$

For the particular case of $\gamma=1$ we substitute (10) and $E_{2,2}\left(-\omega_{0}^{2} t^{2}\right)=\left(1 / \omega_{0} t\right) \sin \omega_{0} t$ on $(21)$ to obtain

$$
\begin{aligned}
q(t ; 1)= & q_{0} \cos \omega_{0} t+\frac{I_{0}}{\omega_{0}} \sin \omega_{0} t \\
& +C V_{0} \sum_{m, n=0}^{\infty} \frac{(-1)^{m+n} \omega_{p}^{2 m} \omega_{0}^{2(n+1)}}{\Gamma[2(n+1)+2 m+1]} t^{2(n+m+1)}
\end{aligned}
$$




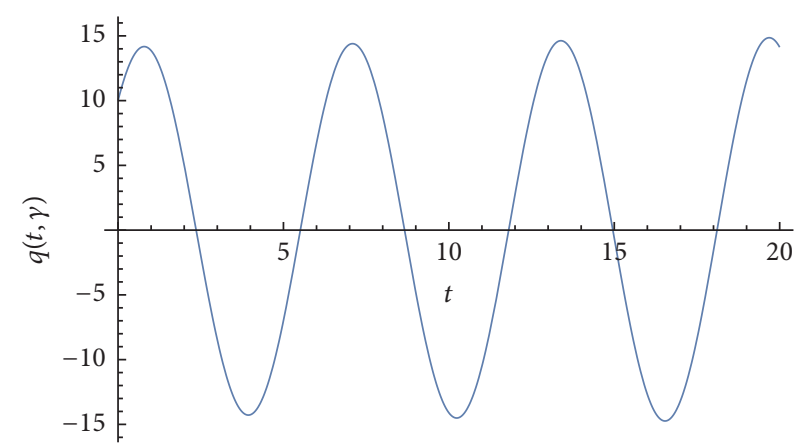

(a)

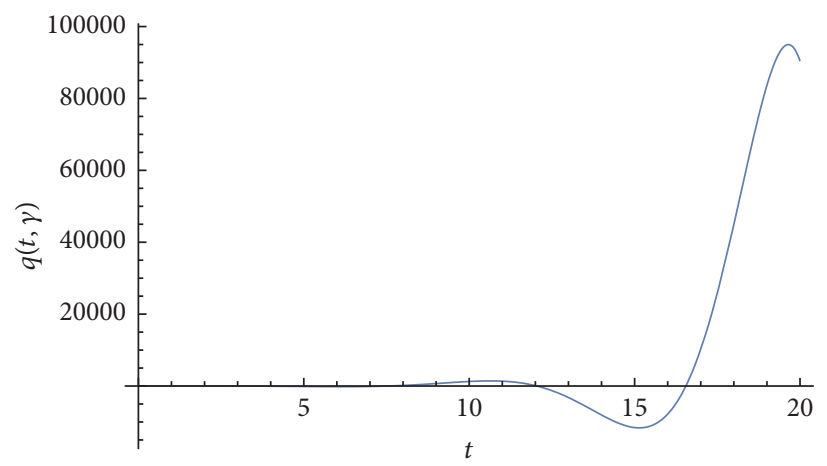

(c)

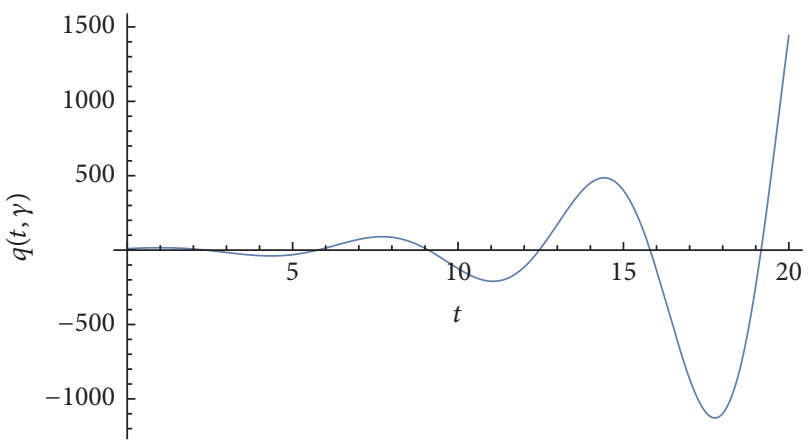

(b)

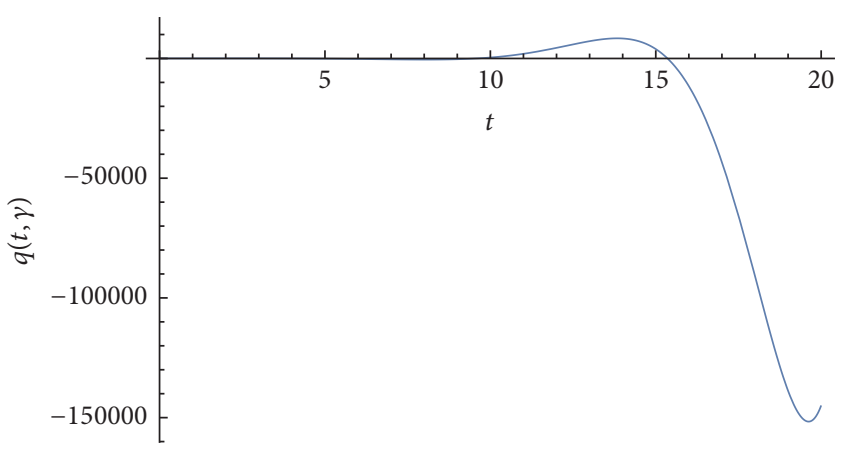

(d)

Figure 2: Simulation of (21) for (a) $\gamma=1$ (classical case); (b) $\gamma=1.3$; (c) $\gamma=1.5$; and (d) $\gamma=2.0$.

TABLE 1: Critical values of $\omega(\gamma)$ for different values of $\gamma$.

\begin{tabular}{lccc}
\hline \multicolumn{4}{c}{ Values of $\omega(\gamma)$} \\
$\gamma$ & Value & $\gamma$ & Value \\
\hline 1 & 1 & 1.1 & 0.990514 \\
1.2 & 0.964193 & 1.3 & 0.924309 \\
1.4 & 0.874075 & 1.5 & 0.816497 \\
1.6 & 0.754272 & 1.7 & 0.689741 \\
1.8 & 0.624859 & 1.9 & 0.561205 \\
1.95 & 0.530233 & 2.0 & 0.50 \\
\hline
\end{tabular}

Taking the same values for $L, C$, and $V_{0}$ as before, plots for different values of $\gamma$ within $1<\gamma \leqslant 2$ are represented in Figures 2(a), 2(b), 2(c), and 2(d).

Table 1 shows the critical values of $\omega(\gamma)$ for different values of $\gamma$.

3.2. RL Electrical Circuit. The fractional differential equation corresponding to a RL circuit with constant source $V_{0}$ is given by [23]

$$
\frac{L}{\sigma^{2(1-\gamma)}} \frac{d^{2 \gamma} q(t)}{d t^{2 \gamma}}+\frac{R}{\sigma^{1-\gamma}} \frac{d^{\gamma} q(t)}{d t^{\gamma}}=V_{0} \quad 1<\gamma \leqslant 2,
$$

and we can rewrite it as

$$
\frac{d^{2 \gamma} q(t)}{d t^{2 \gamma}}+A \frac{d^{\gamma} q(t)}{d t^{\gamma}}=B
$$

where $A=(R / L) \sigma^{1-\gamma}$ and $B=\left(V_{0} / L\right) \sigma^{2(1-\gamma)}$. Using the initial conditions $q(0)=q_{0}$ and $\dot{q}(0)=I_{0}$ and applying the direct Laplace transform, we have

$$
\begin{gathered}
s^{2 \gamma} Q(s)-q_{0} s^{2 \gamma-1}-I_{0} s^{2 \gamma-2}+A s^{\gamma} Q(s)-A q_{0} s^{\gamma-1} \\
-A I_{0} s^{\gamma-2}=\frac{B}{s} .
\end{gathered}
$$

Solving for $Q(s)$, we have

$$
Q(s)=\frac{q_{0}}{s}+\frac{I_{0}}{s^{2}}+\frac{B}{s^{\gamma+1}\left(s^{\gamma}+A\right)} .
$$

For the last terms in (26) we take the highest power of $s$ as a common factor from the denominator and then we expand in an alternating geometric series [38]; given that

$$
\gamma=\frac{R}{L} \sigma
$$

then $A=(R / L)^{\gamma} \gamma^{1-\gamma}$ and $B=\left(V_{0} L / R^{2}\right)(R / L)^{2 \gamma} \gamma^{2(1-\gamma)}$. Substituting these expressions in the inverse Laplace transform, the solution is

$$
q(\tilde{t} ; \gamma)=q_{0}+I_{0} \frac{\tilde{t}}{t}+\frac{V_{0} L}{R^{2}} \sum_{n=0}^{\infty} \frac{(-1)^{n} \gamma^{(n+2)(1-\gamma)}}{\Gamma[\gamma(n+2) \gamma+1]} \widetilde{t}^{(n+2) \gamma}
$$

Consider the electrical circuit RL with $R=100 \Omega, L=$ $10 \mathrm{H}$, and $V_{0}=10$ volts. Plots for values of $\gamma$ within $1<\gamma \leqslant 2$ are represented in Figures 3(a), 3(b), 3(c), and 3(d). 


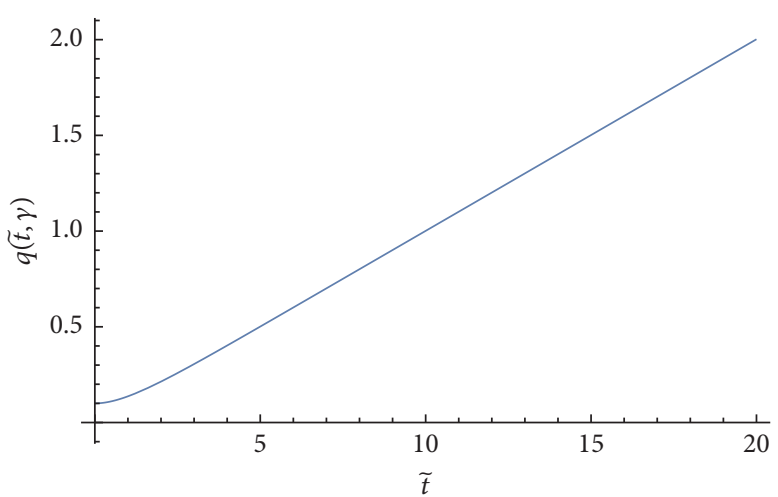

(a)

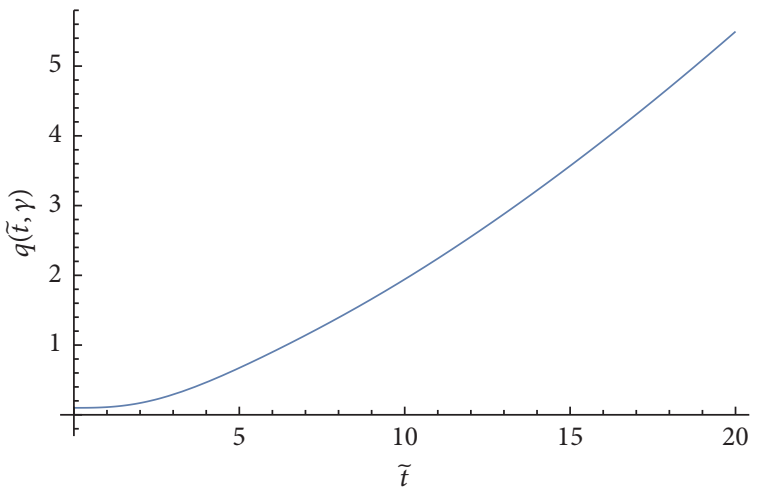

(c)

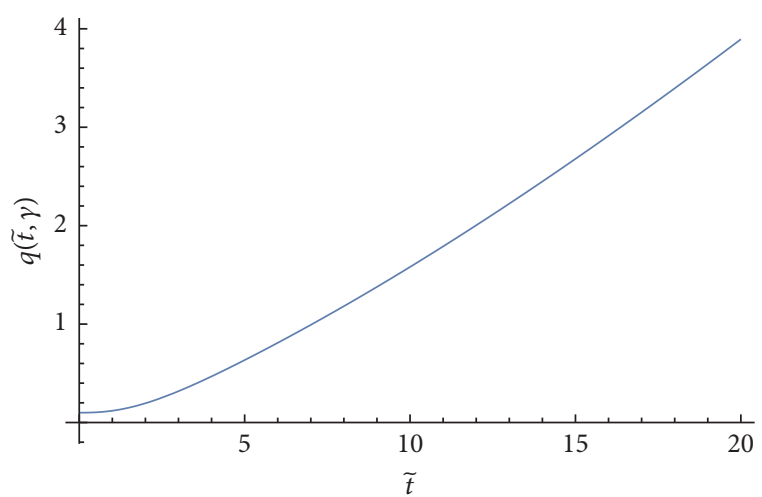

(b)

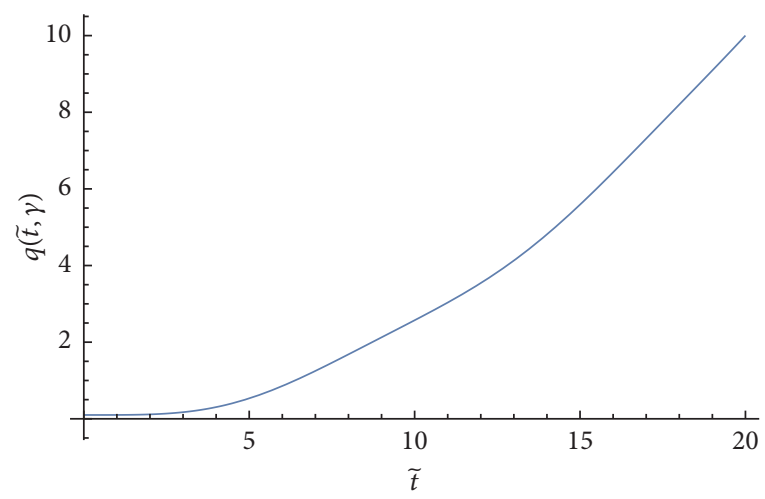

(d)

Figure 3: Simulation of (28) for (a) $\gamma=1$ (classical case); (b) $\gamma=1.3$; (c) $\gamma=1.5$; and (d) $\gamma=2.0$.

Considering an alternating source we have

$$
\frac{L}{\sigma^{2(1-\gamma)}} \frac{d^{2 \gamma} q(t)}{d t^{2 \gamma}}+\frac{R}{\sigma^{1-\gamma}} \frac{d^{\gamma} q(t)}{d t^{\gamma}}=V_{0} \cos (\omega t)
$$

$$
1<\gamma \leqslant 2 \text {, }
$$

rewriting

$$
\frac{d^{2 \gamma} q(t)}{d t^{2 \gamma}}+A \frac{d^{\gamma} q(t)}{d t^{\gamma}}=B \cos (\omega t)
$$

where $A$ and $B$ are the same defined for the RL circuit with constant source. Using the initial conditions $q(0)=q_{0}$ and $\dot{q}(0)=I_{0}$ and applying the direct Laplace transform, we have

$$
Q(s)=\frac{q_{0}}{s}+\frac{I_{0}}{s^{2}}+B \frac{s}{\left(s^{2 \gamma}+A s^{\gamma}\right)\left(s^{2}+\omega^{2}\right)} .
$$

For the last term in (31) we take the highest power of $s$ as a common factor from the denominator and then we expand in an alternating geometric series [38]; then we apply the inverse Laplace transform

$$
\begin{aligned}
& q(t ; \gamma)=q_{0}+I_{0} t+\frac{V_{0} L}{R^{2}} \\
& \quad \cdot \sum_{m, n=0}^{\infty} \frac{(-1)^{m+n}(R / L)^{(m+2) \gamma} \gamma^{(m+2)(1-\gamma)} \omega^{2 n}}{\Gamma[(m+2) \gamma+2 n+1]} t^{(m+2) \gamma+2 n}
\end{aligned}
$$

here, we used relation (27) and the aforementioned expressions defining $A$ and $B$.

Taking the same values for $R, L$, and $V_{0}$, plots for values of $\gamma$ within $1<\gamma \leqslant 2$ are represented in Figures 4(a), 4(b), 4(c), and 4(d).

For both simulations, the numerical solutions show a change in the amplitude of the charge and variations in the phase exhibits fractality in time to different scales and shows the existence of heterogeneities in the electrical components (resistance, inductance). These behaviors depend on the fractional derivative order and modified the constant time of the electrical circuits. The numerical solution exhibits temporal fractality at different scales as well as the existence of material heterogeneities in the electrical components.

Table 2 shows different values of the constant time $\tau=$ $(L / R)^{\gamma} \gamma^{1-\gamma}$ for arbitrary values of $\gamma$.

\section{Conclusions}

In this paper, we studied the behavior of third- and fourthorder differential equations modeling LC and RL electrical circuits. These high order equations are naturally obtained using fractional calculus for derivative orders chosen in the interval [1,2). Results show that the LC electrical circuit behavior is strongly dependent on the fractional order, which was varied within the interval $[1,2)$ and determines the frequency according to (15). The fractional time constant 


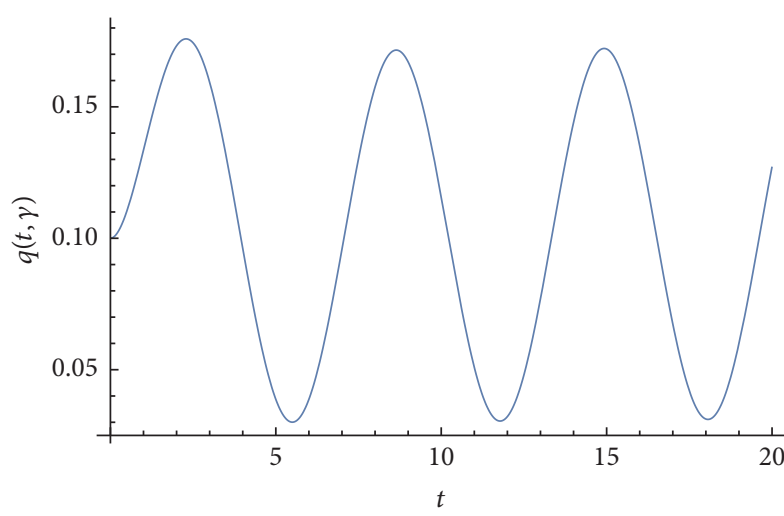

(a)

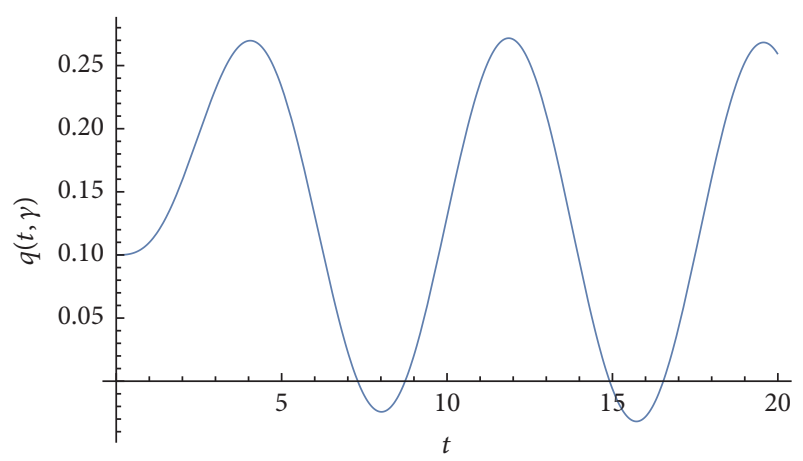

(c)

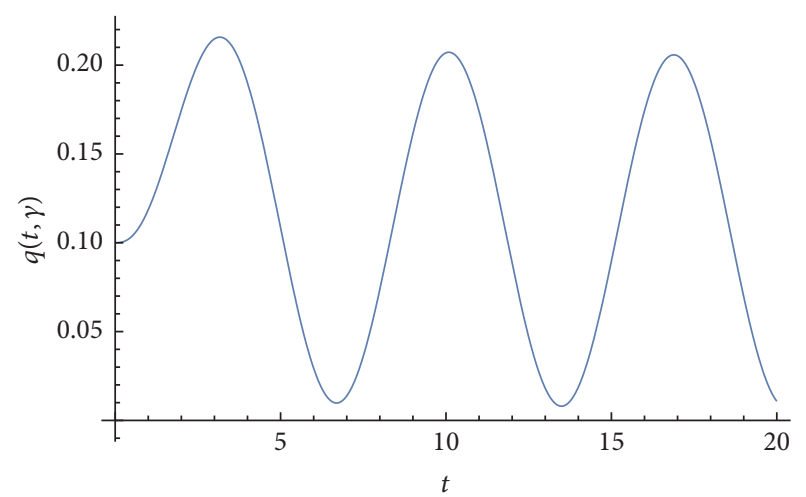

(b)

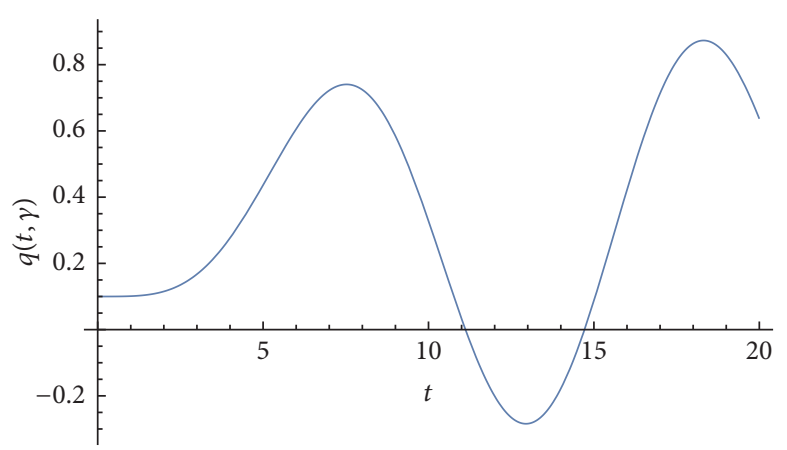

(d)

Figure 4: Simulation of (32) for (a) $\gamma=1$ (classical case); (b) $\gamma=1.3$; (c) $\gamma=1.5$; and (d) $\gamma=2.0$.

TABLE 2: Values of the constant time $\tau=(L / R)^{\gamma} \gamma^{1-\gamma}$ for arbitrary values of $\gamma$.

\begin{tabular}{lccc}
\hline \multicolumn{4}{c}{ Values of constant time $\tau$} \\
$\gamma$ & Value & $\gamma$ & Value \\
\hline 1 & 0.1 & 1.1 & 0.0786793 \\
1.2 & 0.0608364 & 1.3 & 0.0463252 \\
1.4 & 0.0347976 & 1.5 & 0.0258199 \\
1.6 & 0.0189465 & 1.7 & 0.0137621 \\
1.8 & 0.00990335 & 1.9 & 0.00706516 \\
1.95 & 0.00594931 & 2.0 & 0.005 \\
\hline
\end{tabular}

$\tau=(L / R)^{\gamma} \gamma^{1-\gamma}$ is modified in the RL electrical circuit with the order $\gamma$ of the fractional derivative. This constant time tends to move forward in time as the exponent $\gamma$ changes the dynamics and the relaxation times of the system (they are faster). For all figures, in the range $(1 ; 2)$ the charge exhibits fractional jerky dynamics; furthermore, when the order of the derivative is equal to 2 the charge exhibits Jounce dynamics. Our results indicate that the fractional order has an important influence on the charge and the general solution of the fractional circuit depends on the parameter $\gamma$. These solutions represent a new family of solutions for the charge. In the range $\gamma \in(1,2)$ the charge is unstable, the charge exhibits an increment of the amplitude, and the behavior becomes anomalously dispersive (the charge increases with increasing order of $\gamma$ ). The electrical circuits studied and their respective analogue mechanical system can be used to analyze the vibration levels of machinery, serial mechanisms, robotics, oscillating circuits modeling, and instability of electrical and mechanical circuits, to evaluate reconfigurable machines or to make mobility analysis or algebraic formulations of motion equations.

\section{Competing Interests}

The authors declare that they have no competing interests.

\section{Authors' Contributions}

All the authors have equally contributed in this paper and read the final version before the submission.

\section{Acknowledgments}

José Francisco Gómez Aguilar acknowledges the support provided by CONACYT: Cátedras CONACYT para Jovenes Investigadores 2014. 


\section{References}

[1] K. B. Oldham and J. Spanier, The Fractional Calculus, Academic Press, New York, NY, USA, 1974.

[2] K. S. Miller and B. Ross, An Introduction to the Fractional Calculus and Fractional Differential Equations, John Wiley \& Sons, New York, NY, USA, 1993.

[3] I. Podlubny, Fractional Differential Equations, Academic Press, New York, NY, USA, 1999.

[4] D. Baleanu, K. Diethelm, E. Scalas, and J. J. Trujillo, Fractional Calculus Models and Numerical Methods, Complexity, Nonlinearity and Chaos, World Scientific, 2012.

[5] S. Westerlund and L. Ekstam, "Capacitor theory," IEEE Transactions on Dielectrics and Electrical Insulation, vol. 1, no. 5, pp. 826-839, 1994.

[6] F. Mainardi, Fractional Calculus and Waves in Linear Viscoelasticity, Imperial College Press, London, UK, 2010.

[7] A. Coronel-Escamilla, J. Gómez-Aguilar, M. López-López, V. Alvarado-Martínez, and G. Guerrero-Ramírez, "Triple pendulum model involving fractional derivatives with different kernels," Chaos, Solitons \& Fractals, vol. 91, pp. 248-261, 2016.

[8] V. V. Uchaikin, Fractional Derivatives for Physicists and Engineers, Springer, Berlin, Germany, 2013.

[9] S. Kim, S.-J. Kim, K. M. Kim et al., "Physical electro-thermal model of resistive switching in bi-layered resistance-change memory," Scientific Reports, vol. 3, article 1680, 2013.

[10] C. H. Henager Jr. and W. T. Pawlewicz, "Thermal conductivities of thin, sputtered optical films," Applied Optics, vol. 32, no. 1, pp. 91-101, 1993.

[11] I. Podlubny, I. Petras, B. M. Vinagre, P. O'Leary, and L. Dorcek, "Analogue realizations of fractional-order controllers," Nonlinear Dynamics, vol. 29, no. 1-4, pp. 281-296, 2002.

[12] I. Petráš, "Fractional-order memristor-based Chua's circuit," IEEE Transactions on Circuits and Systems II: Express Briefs, vol. 57, no. 12, pp. 975-979, 2010.

[13] J. F. Gómez-Aguilar, "Behavior characteristics of a cap-resistor, memcapacitor, and a memristor from the response obtained of $\mathrm{RC}$ and RL electrical circuits described by fractional differential equations," Turkish Journal of Electrical Engineering \& Computer Sciences, vol. 24, no. 3, pp. 1421-1433, 2016.

[14] A. S. Elwakil, "Fractional-order circuits and systems: an emerging interdisciplinary research area," IEEE Circuits and Systems Magazine, vol. 10, no. 4, pp. 40-50, 2010.

[15] I. Petras, Fractional-Order Nonlinear Systems: Modeling, Analysis and Simulation, Springer, Berlin, Germany, 2011.

[16] H. Samavati, A. Hajimiri, A. R. Shahani, G. N. Nasserbakht, and T. H. Lee, "Fractal capacitors," IEEE Journal of Solid-State Circuits, vol. 33, no. 12, pp. 2035-2041, 1998.

[17] S. I. R. Arias, D. R. Muñoz, J. S. Moreno, S. Cardoso, R. Ferreira, and P. J. P. Freitas, "Fractional modeling of the AC largesignal frequency response in magnetoresistive current sensors," Sensors, vol. 13, no. 12, pp. 17516-17533, 2013.

[18] A. M. A. El-Sayed, H. M. Nour, W. E. Raslan, and E. S. ElShazly, "Fractional parallel RLC circuit," Alexandria Journal of Mathematics, vol. 3, no. 1, pp. 11-23, 2012.

[19] A. G. Radwan and K. N. Salama, "Fractional-order $R C$ and $R L$ circuits," Circuits, Systems and Signal Processing, vol. 31, no. 6, pp. 1901-1915, 2012.
[20] R. Caponetto, G. Dongola, G. Maione, and A. Pisano, "Integrated technology fractional order proportional-integral-derivative design," Journal of Vibration and Control, vol. 20, no. 7, pp. 1066-1075, 2014.

[21] A. Obeidat, M. Gharaibeh, M. Al-Ali, and A. Rousan, "Evolution of a current in a resistor," Fractional Calculus and Applied Analysis, vol. 14, no. 2, pp. 247-259, 2011.

[22] J. F. Gómez-Aguilar, J. J. Rosales-García, J. J. Bernal-Alvarado, T. Córdova-Fraga, and R. Guzmán-Cabrera, "Fractional mechanical oscillators," Revista Mexicana de Fisica, vol. 58, no. 4, pp. 348-352, 2012.

[23] F. Gómez, J. Rosales, and M. Guía, "RLC electrical circuit of non-integer order," Central European Journal of Physics, vol. 11, no. 10, pp. 1361-1365, 2013.

[24] M. Guía, F. Gómez, and J. Rosales, "Analysis on the time and frequency domain for the RC electric circuit of fractional order," Central European Journal of Physics, vol. 11, no. 10, pp. 1366-1371, 2013.

[25] J. F. Gómez-Aguilar, T. Córdova-Fraga, J. E. Escalante-Martínez, C. Calderón-Ramón, and R. F. Escobar-Jiménez, "Electrical circuits described by a fractional derivative with regular kernel," Revista Mexicana de Física, vol. 62, no. 2, pp. 144-154, 2016.

[26] F. Gómez-Aguilar, R. Razo-Hernández, J. Rosales-García, and M. Guía-Calderón, "Fractional RC and LC electrical circuits," Revista de Ingeniería, Investigación y Tecnología, UNAM, vol. 2, pp. 311-319, 2014.

[27] J. F. Gómez-Aguilar and D. Baleanu, "Fractional transmission line with losses," Zeitschrift fur Naturforschung, vol. 69, no. 1011, pp. 539-546, 2014.

[28] S. H. Schot, "Jerk: the time rate of change of acceleration," American Journal of Physics, vol. 46, no. 11, pp. 1090-1094, 1978.

[29] J. D. Smith, "Vibration monitoring of bearings at low speeds," Tribology International, vol. 15, no. 3, pp. 139-144, 1982.

[30] S. Lahdelma, "New vibration severity evaluation criteria for condition monitoring," Research Report 85, University of Oulu, 1992.

[31] S. Lahdelma, On the higher order derivatives in the laws of motion and their application to an active force generator and to condition monitoring [DSc Tech thesis], University of Oulu, Oulu, Finland, Research report no. 101, 1995.

[32] S. Lahdelma, J. Strackeljan, and D. Behr, "Combination of higher order derivatives and a fuzzy classifier as a new approach for monitoring rotating machinery," in Proceedings of the 12th International Congress on Condition Monitoring and Diagnostic Engineering Management, University of Sunderland, 1999.

[33] B. T. Krishna, "Studies on fractional order differentiators and integrators: a survey," Signal Processing, vol. 91, no. 3, pp. 386426, 2011.

[34] A. G. Radwan and K. N. Salama, "Passive and active elements using fractional $L_{\beta} C_{\alpha}$ circuit," IEEE Transactions on Circuits and Systems I: Regular Papers, vol. 58, no. 10, pp. 2388-2397, 2011.

[35] A. G. Radwan, A. M. Soliman, and A. S. Elwakil, "Design equations for fractional-order sinusoidal oscillators: four practical circuit examples," International Journal of Circuit Theory and Applications, vol. 36, no. 4, pp. 473-492, 2008.

[36] X.-X. Xu, S.-J. Ma, and P.-T. Huang, "New concepts in electromagnetic jerky dynamics and their applications in transient processes of electric circuit," Progress in Electromagnetics Research M, vol. 8, pp. 181-194, 2009. 
[37] D. Shantanu and N. C. Pramanik, "Micro structural roughness of electrodes manifesting as temporal fractional order differential equation in super-capacitor transfer characteristics," International Journal of Mathematics \& Computation, vol. 20, no. 3, pp. 94-113, 2013.

[38] F. Y. Alzoubi, M. K. Alqadi, H. M. Al-Khateeb, S. M. Saadeh, and N. Y. Ayoub, "Solution of a fractional undamped forced oscillator," Jordan Journal of Physics, vol. 5, no. 3, pp. 129-134, 2012. 


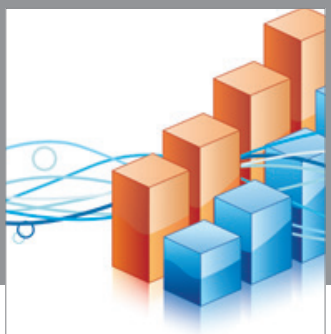

Advances in

Operations Research

vatem alat4

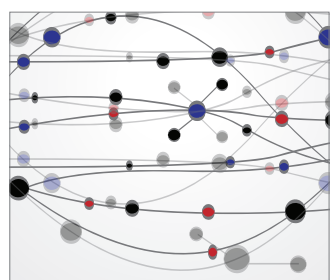

\section{The Scientific} World Journal
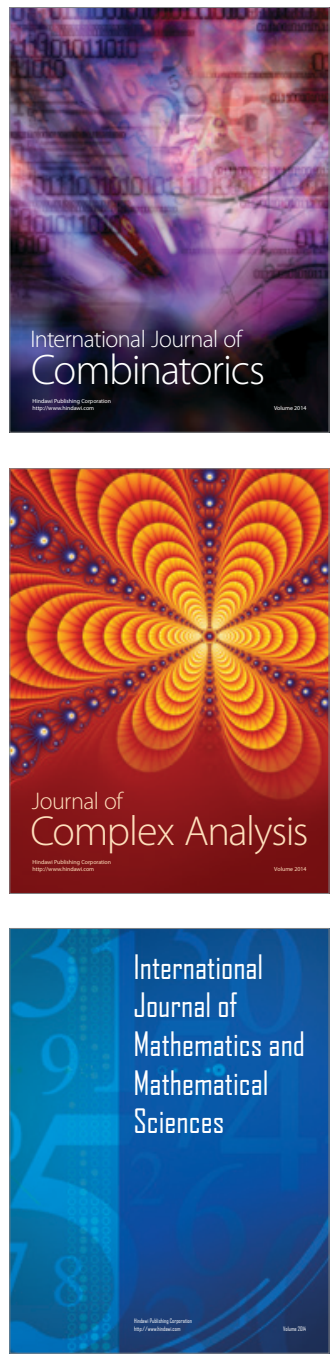
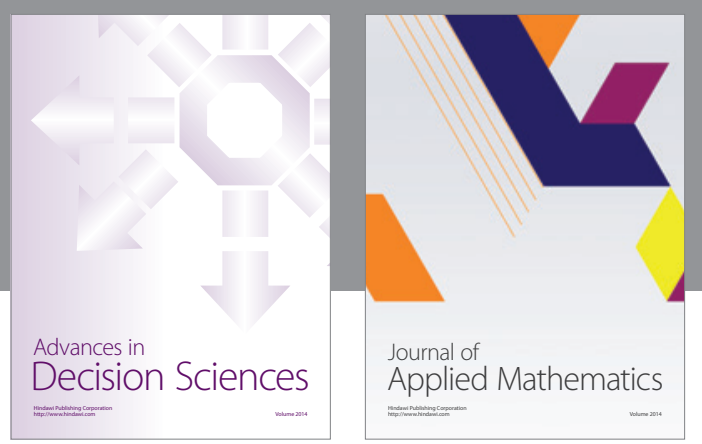

Algebra

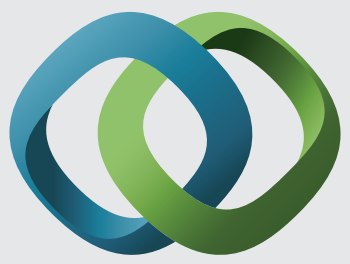

\section{Hindawi}

Submit your manuscripts at

http://www.hindawi.com
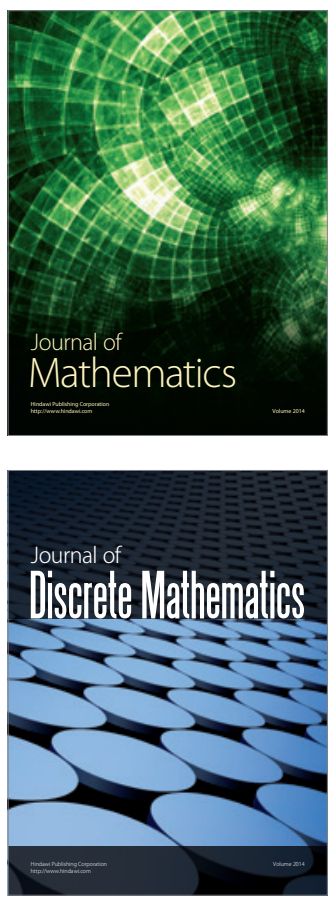

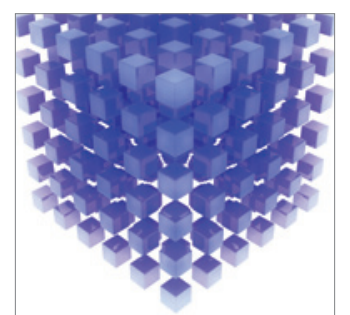

Mathematical Problems in Engineering
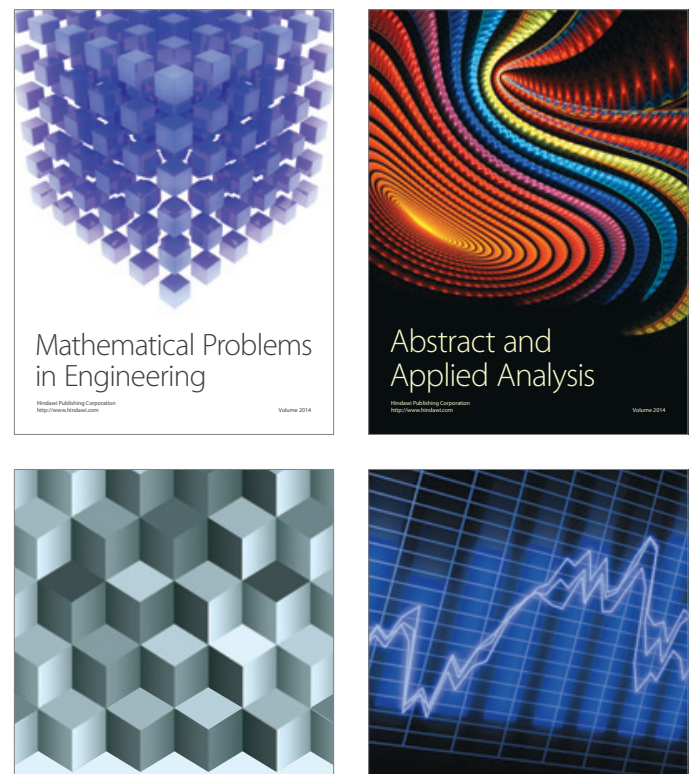

Journal of

Function Spaces

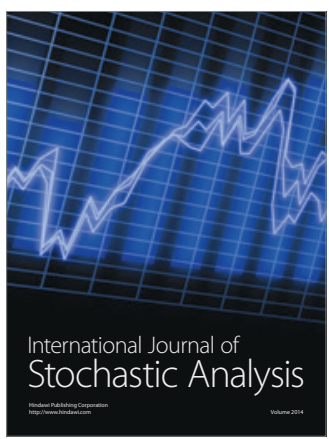

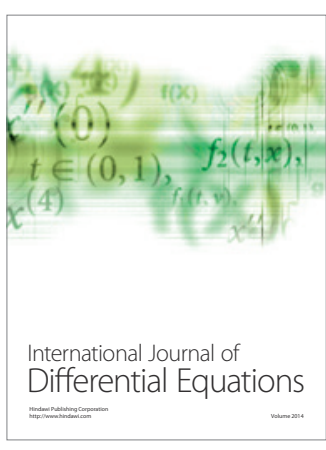
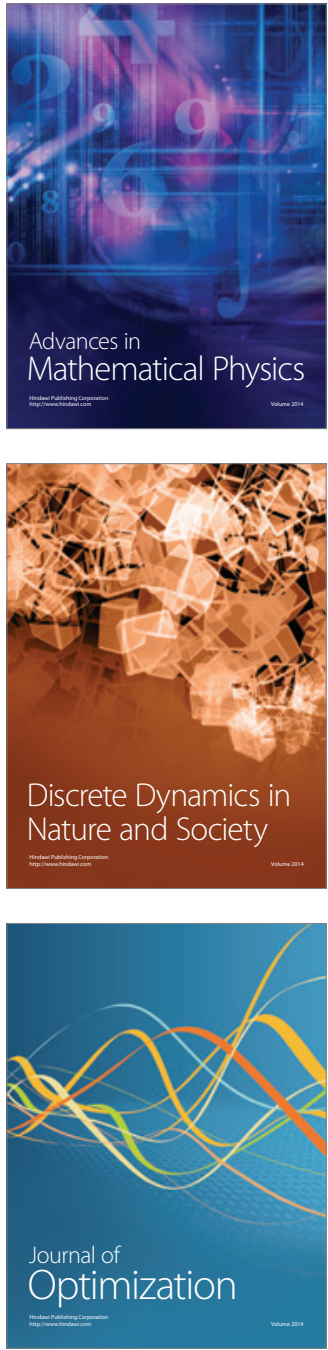